Original Article

\title{
IN SILICO STUDIES ON DIACYL DERIVATIVES OF PHLOROGLUCINOL TO ENHANCE PHARMACODYNAMIC AND PHARMACOKINETIC PROFILES OF 2,4,6-TRIHYDROXY-3- GERANYL-ACETOPHENONE
}

\author{
SHEAU WEI CHIONG ${ }^{1}$, CHEAN HUI NG ${ }^{1 *}$, KHOZIRAH SHAARI ${ }^{2,3}$
}

${ }^{1}$ School of Pharmacy, Management and Science University, Shah Alam 40100, Malaysia, ${ }^{2}$ Laboratory of Natural Medicines and Products (NaturMeds), Institute of Bioscience, Universiti Putra Malaysia, Serdang 43400, Malaysia, ${ }^{3}$ Department of Chemistry, Faculty of Science, Universiti Putra Malaysia, Serdang 43400, Malaysia *Email: chng@msu.edu.my

Received: 10 Dec 2020, Revised and Accepted: 15 Jan 2021

\begin{abstract}
Objective: The purpose of this study was to evaluate the LOX inhibitory activity, and predict the drug likeness properties of designed diacyl derivatives of phloroglucinol, using in silico method.

Methods: The designed derivatives were subjected to molecular docking using AUTODOCK while the receptor used in this study was built from SWISS MODEL. Drug likeness properties of the derivatives were calculated by online programs i.e. MOLINSPIRATION and PreADMET.

Results: Molecular docking study revealed that designed tHGA derivative with four-carbon chain length exhibited the best binding affinity with the docking scores of $-7.26 \mathrm{kcal} / \mathrm{mol}$. Three types of binding interactions were observed between the derivatives and the receptor site i.e H-bonding, hydrophobic and Van der Waals interactions. The important amino acid residues involved in H-bonding were Gln495 and Gln697, while other amino acid residues, such as Leu754 and Ile 553, were involved in the Van der Waals interaction. The designed tHGA derivatives were mainly stabilized through hydrophobic interactions with His499, His504, Ile538, Phe557 and Val750. In silico physicochemical calculations predicted that all the designed derivatives passed the Lipinski's Rule of 5, and have good human intestinal absorption property (HIA $>70 \%$ ). Further, all the designed derivatives showed moderate central nervous system absorption $(0.6<\mathrm{BBB}<2.0)$, except for the derivative with a longer $(5-\mathrm{Cs})$ chain length.
\end{abstract}

Conclusion: The findings of the present study suggested that changing the acyl and geranyl side chains of the natural product molecule, tHGA, into two acyl bearing side chains, will improve its pharmacodynamic and pharmacokinetic profiles.

Keywords: 2,4,6-trihydroxy-3-geranylacetophenone, Lipoxygenase, AUTODOCK, SWISS MODEL, Drug likeness properties

(C) 2021 The Authors. Published by Innovare Academic Sciences Pvt Ltd. This is an open access article under the CC BY license (https://creativecommons.org/licenses/by/4.0/) DOI: https://dx.doi.org/10.22159/ijap.2021v13i2.40784. Journal homepage: https://innovareacademics.in/journals/index.php/ijap

\section{INTRODUCTION}

Asthma is a chronic disease characterized by recurrent attacks of breathlessness and wheezing, which differ in severity and frequency among different individuals [1]. Human 5-lipoxygenase (5-LO) plays a crucial role in pathogenesis of asthma through the metabolism of arachidonic acid (AA) at the 5-position which leads to the formation of 5-hydroperoxyeicosatetraenoic acid (5-HPETE). 5-HPETE is then converted into leukotrienes $\mathrm{LTA}_{4}$ which is then enzymatically metabolized to $\mathrm{LTB}_{4}, \mathrm{LTC}_{4}, \mathrm{LTD}_{4}$, and $\mathrm{LTE}_{4}$. The lipid mediators cysteinyl leukotrienes (CysLTs) including $\mathrm{LTC}_{4}, \mathrm{LTD}_{4}$, and $\mathrm{LTE}_{4}$ are the major source of potent proinflammatory mediators that bind to $\mathrm{H}$ protein-coupled receptors (GPCRs), contributing to allergic disorders, such as asthma [2-4].

Meanwhile, the human 15-LOX-1 enzyme converts arachidonic acid (AA) and linoleic acid into 15(S)-HPETE and 13(S)hydroperoxyoctadecadienoic acid (13(S)-HPODE) which subsequently undergo reduction and transformation into $15(S)$ hydroxyeicosatetraenoic acid (15(S)-HETE) and 13(S)hydroxyoctadecadienoic acid (13(S)-HODE). Both 15(S)-HETE and 13(S)-HODE metabolites cause epithelial injury in the airway, resulting in asthma [5].

Plant LOX is also involved in the oxygenation of linoleic acid to generate 13-HODE [6]. Therefore, drug design strategies targeting the inhibition of the key enzyme that responsible for biosynthesis of pro-inflammatory metabolites mentioned earlier, are crucial for treating asthma. Nevertheless, soybean LOX (15sLOX) has been used as an alternative biological screen, replacing human LOX, due to difficulties in obtaining purified human LOX and the highly conserved catalytic domain of both isoenzymes [7-9].

The natural product molecule 2,4,6-trihydroxy-3-geranyl acetophenone (tHGA), isolated from Melicope ptelefolia has been reported to inhibit the synthesis of cysteinyl leukotrienes (CysLTs) in activated macrophages through 5-LOX inhibition [10]. Previous animal study by Ismail et al., (2012) also confirmed the effectiveness of tHGA as a potential lead compound in preventing allergic airway inflammation [11]. Following this, a series of tHGA analogues were designed and synthesized by $\mathrm{Ng}$ et al., (2014), by employing FriedelCraft acylation and alkylation reactions. They further investigated the structure-activity relationship of the analogues, based on the soybean 15-LOX enzyme inhibitory activity, where the analogues were shown to exhibit potent, dose-dependent activity, with $\mathrm{IC}_{50}$ value of 10.31-27.61 $\mu \mathrm{M}$. The most active tHGA analogue exhibited 2-fold higher 15-LOX inhibitory activity than tHGA ( $\mathrm{IC}_{50}=23.61 \mu \mathrm{M}$ ). The findings strongly suggested that elongation of the acyl-bearing aliphatic chain (2Cs-5Cs) and presence of the aromatic moiety, greatly enhanced the enzyme inhibition in comparison to the unmodified structure of tHGA. However, increase the chain length of the molecules is expected to reduce solubility, and, therefore, will also indirectly affect the bioavailability of the compound $[12,13]$. Thus, in-sillico studies are important to give a better insight into the structure-activity relationship (SAR) of designed molecules, in order to justify the full picture of the pharmacophore. Therefore, this study aimed to investigate the potential LOX inhibition property and predict the drug likeness properties of several designed derivatives using in silico method.

\section{METHODS}

\section{Preparation of protein and ligand molecules}

Homology model of soybean LOX-1, built using SWISS MODEL, was retrieved from our previous study [13] and used in the present work Chem Draw Ultra 12.0 was used to construct 3-dimensional structures of the target compounds [14]. The chemical structures of tHGA and the most active tHGA analogue were obtained from a previous study [12], while the designed tHGA derivatives (1-4) are shown in fig. 1. 


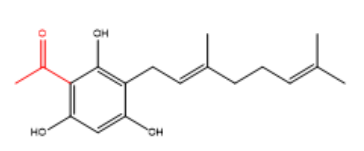

tHGA

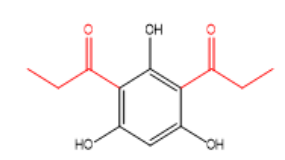

2,4-dipropionylphloroglucinol (Diacyl derivative 1)

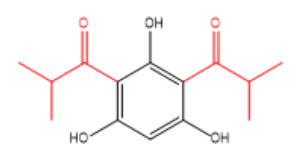

2,4-di-isobutyrlphloroglucinol

(Diacyl derivative 3 )

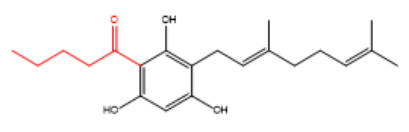

tHGA analogue

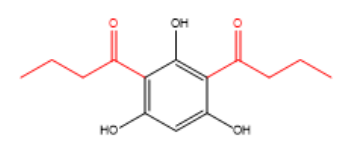

2,4-dibutyrylphloroglucinol (Diacyl derivative 2)

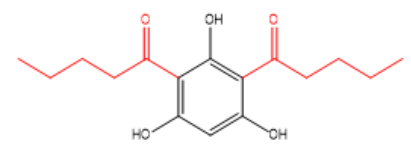

2,4-dipentanoylphloroglucinol

(Diacyl derivative 4)

Fig. 1: Chemical structures for designed derivatives, tHGA and its most active analogue

\section{Molecular docking}

Docking study was applied to the modelled soybean LOX-1 receptor using Autodock tool (ADT) software. The protein needs to be pretreated before docking by getting rid of water and co-crystallized inhibitors, excluding the cofactor iron $\left(\mathrm{Fe}^{3+}\right)$. The Autodock tool was used to assign Gasteiger charges, adding missing hydrogen atoms, and non-polar hydrogen atoms were merged. The molecular docking calculations were carried out on the active site of the modelled soybean LOX-1. The grid box $(24.958 \mathrm{x}-0.033 \mathrm{x} 19.021 \AA$ and $100 \mathrm{x}$ $100 \times 100 \AA$ dimensions) were centered on the co-crystallized inhibitor. The Lamarckian Genetic Algorithm (LGA) was employed and a total of 100 calculations were performed. All other parameters were set at their default values. The binding affinity of the derivatives was evaluated through interpolated energy calculations (calculated free energy of binding). The best docked ligands were quantified through scoring function. The analysis of the binding interaction was examined through Discovery Studio (DS) Viewer software.

\section{Prediction of drug likeness properties}

Nine descriptors were calculated through Molinspiration online database (www. molinspiration. com), including molecular formula, molecular weight, LogP, polar surface area, number of $\mathrm{O}$ or $\mathrm{N}$ atom, number of $\mathrm{OH}$ or $\mathrm{NH}$ atom, number of rotatable bonds, volume, and number of violations to Lipinski's rule, for all selected compounds $[15,16]$.

The Pre-ADMET program was accessed at http://preadmet.bmdrc.org/. The chemical structures of selected compounds were drawn and analyzed automatically by the program. The program automatically calculates predictive plasma protein binding (PPB), blood brain barrier (BBB) permeability, human intestinal absorption, permeability for Caco2-cell, MDCK cell, and skin permeability values [17].

\section{RESULTS}

\section{Molecular docking}

The four designed diacyl derivatives (1-4) of tHGA, along with tHGA and the previously reported most active analogue were subjected to molecular docking onto the active LOX receptor site, using Autodock. The molecular docking energy of the target compounds are tabulated in table 1. Derivative 2 showed the highest docking score $(-7.26$ $\mathrm{kcal} / \mathrm{mol})$, followed by tHGA $(-6.76 \mathrm{kcal} / \mathrm{mol})$, tHGA analogue $(-6.71$ $\mathrm{kcal} / \mathrm{mol})$, derivative $4(-6.68 \mathrm{kcal} / \mathrm{mol})$, derivative $1(-6.37 \mathrm{kcal} / \mathrm{mol})$ and derivative 3 with $(-6.26 \mathrm{kcal} / \mathrm{mol})$. Derivative 3 has the lowest docking score among all four diacyl derivatives. This could be due to the bulkier acyl side chain, which causes more stearic hindrance, even though the binding pattern is similar to tHGA[18]. The molecular binding model of diacyl derivatives (1-4), tHGA and tHGA analogue in the active site of LOX-1 homology model are shown in fig. 2-7.

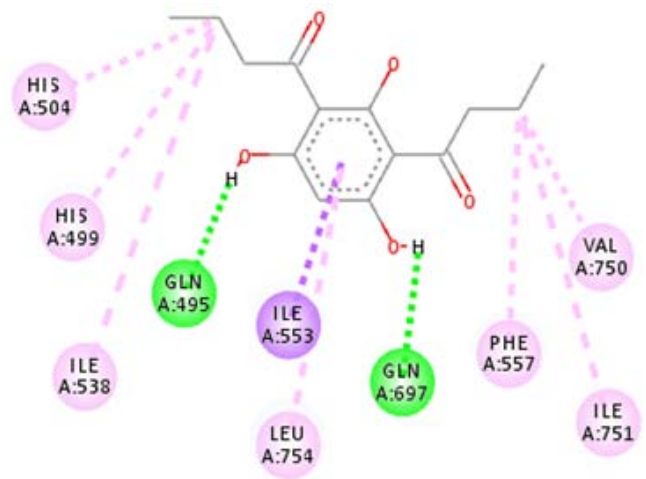

(a)

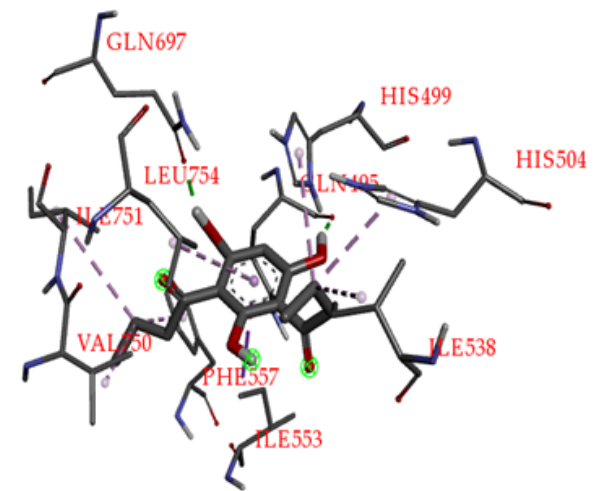

(b)

Fig. 2: Molecular binding model of derivative 2 in the active site of LOX-1 homology model: (a) 2D diagram; (b) 3D diagram. In (a), green line indicates hydrogen-bonding interactions, light purple line indicates alkyl and $\pi$-alkyl interactions, purple line indicates $\pi$-sigma interactions. In (b), the atoms are colored gray for carbon, red for oxygen, blue for nitrogen, and light grey for hydrogen; amino acid residues are indicated in red color word 
Table 1: Molecular docking energy of diacyl derivatives (1-4), tHGA and most active tHGA analogue

\begin{tabular}{ll}
\hline Compound & Binding energy (kcal/mol) \\
\hline 1 & -6.37 \\
2 & -7.26 \\
3 & -6.26 \\
4 & -6.68 \\
tHGA & -6.76 \\
tHGA analogue & -6.71 \\
\hline
\end{tabular}

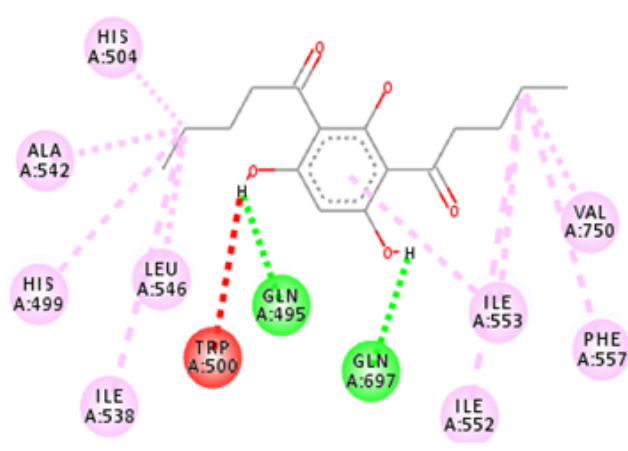

(a)

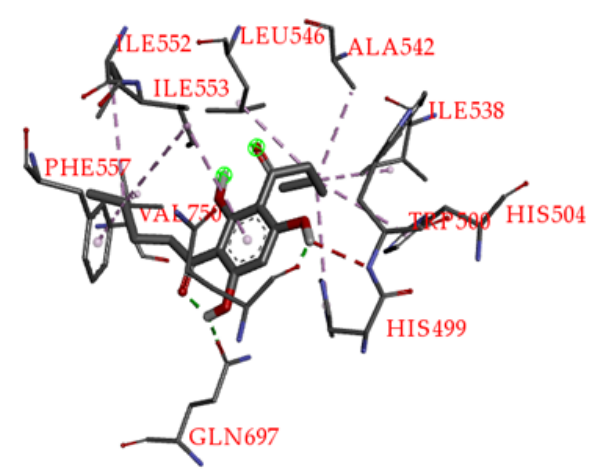

(b)

Fig. 3: Molecular binding model of derivative 4 in the active site of LOX-1 homology model: (a) 2D diagram; (b) 3D diagram. In (a), green line indicates hydrogen-bonding interactions, light purple line indicates alkyl and $\pi$-alkyl interactions, red line indicates unfavorable donor-donor interactions. In (b), the atoms are colored gray for carbon, red for oxygen, blue for nitrogen, and light grey for hydrogen; amino acid residues are indicated in red color word

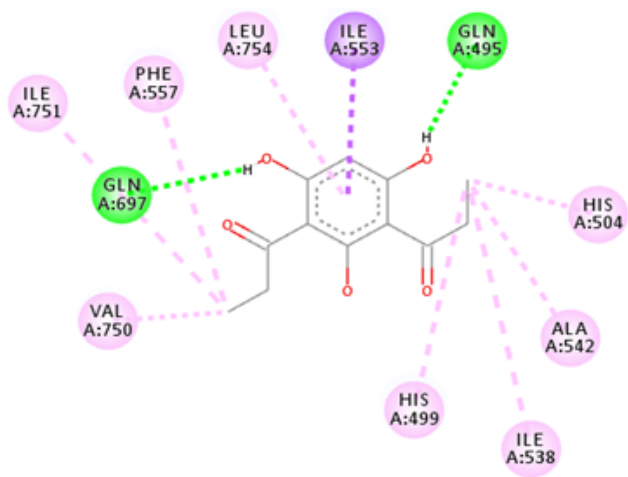

(a)

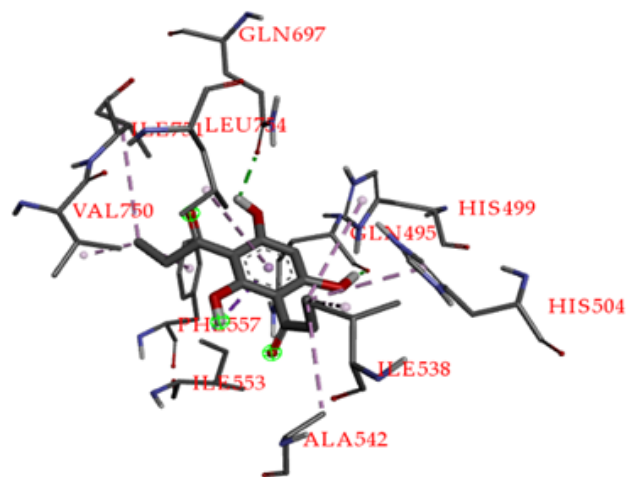

(b)

Fig. 4: Molecular binding model of derivative 1 in the active site of LOX-1 homology model: (a) 2D diagram; (b) 3D diagram. In (a), green line indicates hydrogen-bonding interactions, light purple line indicates alkyl and $\pi$-alkyl interactions, purple line indicates $\pi$-sigma interactions. In (b), the atoms are colored gray for carbon, red for oxygen, blue for nitrogen, and light grey for hydrogen; amino acid residues are indicated in red color word

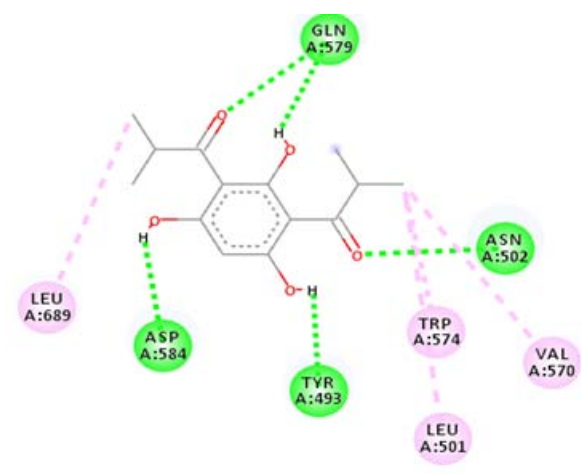

(a)

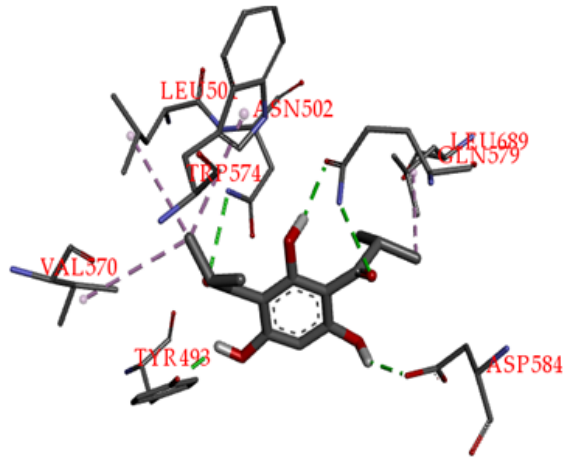

(b)

Fig. 5: Molecular binding model of derivative 3 in the active site of LOX-1 homology model: (a) 2D diagram; (b) 3D diagram. In (a), green line indicates hydrogen-bonding interactions, light purple line indicates alkyl and $\pi$-alkyl interactions. In (b), the atoms are colored gray for carbon, red for oxygen, blue for nitrogen, and light grey for hydrogen; amino acid residues are indicated in red color word 


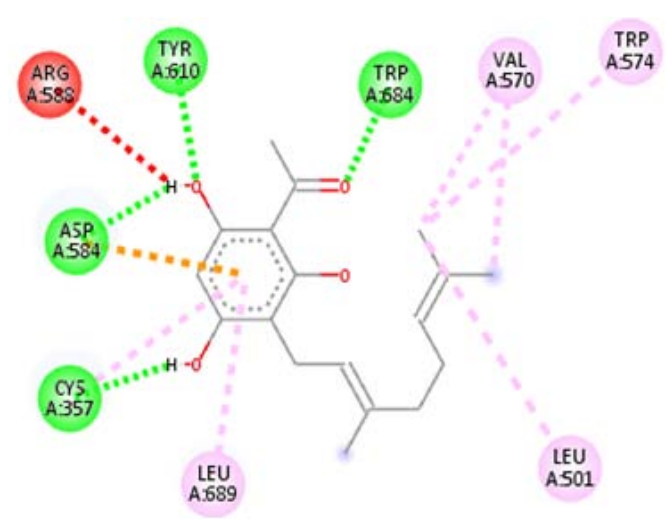

(a)

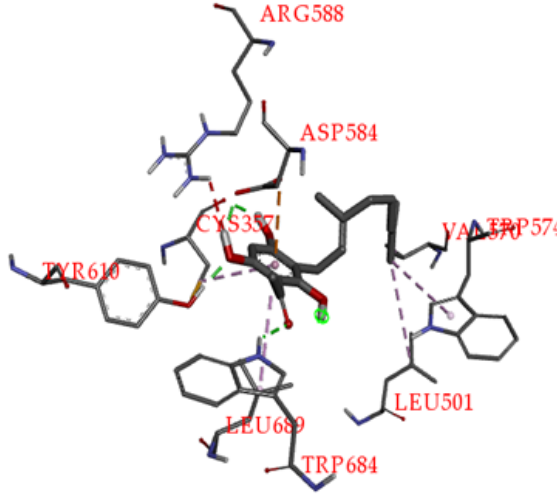

(b)

Fig. 6: Molecular binding model of tHGA in the active site of LOX-1 homology model: (a) 2D diagram; (b) 3D diagram. In (a), green line indicates hydrogen-bonding interactions, light purple line indicates alkyl and $\pi$-alkyl interactions, orange line indicates $\pi$-anion interaction, red line indicates unfavorable hydrogen donor-donor interaction. In (b), the atoms are colored gray for carbon, red for oxygen, blue for nitrogen, and light grey for hydrogen; amino acid residues are indicated in red color word

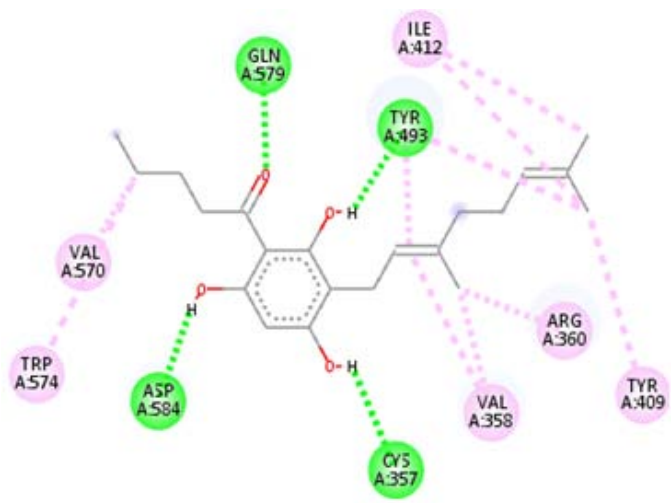

(a)

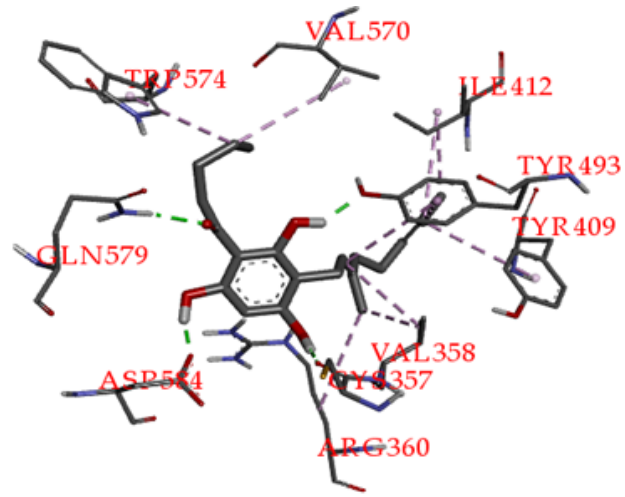

(b)

Fig. 7: Molecular binding model of the most potent tHGA analogue in the active site of LOX-1 homology model: (a) 2D diagram; (b) 3D diagram. In (a), green line indicates hydrogen-bonding interactions, light purple line indicates alkyl and $\pi$-alkyl interactions. In (b), the atoms are colored gray for carbon, red for oxygen, blue for nitrogen, and light grey for hydrogen; amino acid residues are indicated in red color word

\section{Prediction of drug likeness properties}

Lipinski's rule of 5 states that a chemical or drug compound is more likely to be a potential drug for human use if it fulfills the following criteria i. e log P value of no more than 5 , number of hydrogen bond acceptors (nON) of no more than 10, number of hydrogen bond donors (nOHNH) of no more than 5 and molecular weight of no more than 500, number of rotatable bonds of no more than 10 and polar surface area (PSA) of no more than $140 \AA$ [19]. Commonly, the number of violations for an oral drug does not exceed one under the given criteria of Lipinski's rule of 5 [19]. The drug likeness and molecular properties of the target compounds in the present study were calculated by using Molinspiration cheminformatics software. The values are shown in table 2. Meanwhile, the prediction ADMET results for all the tested compounds are shown in table 3. Caco-2 permeability, MDCK permeability, HIA and SKlogP values were calculated based on in vitro models used to assess the intestinal absorption of a compound [20].

Table 2: Molinspiration property of diacyl derivatives (1-4), tHGA and most active tHGA analogue

\begin{tabular}{|c|c|c|c|c|c|c|c|c|c|c|}
\hline Compound & $\begin{array}{l}\text { Molecular } \\
\text { formula }\end{array}$ & $\begin{array}{l}\text { Molecular } \\
\text { weight }\end{array}$ & $\begin{array}{l}\text { Mi Log } \\
\text { Pc }\end{array}$ & TPSA $^{\mathbf{b}}$ & nON ${ }^{d}$ & nOHNHe & nrotb $^{f}$ & Volume & $\begin{array}{l}\text { No. } \\
\text { Violation }\end{array}$ & $\begin{array}{l}\text { In silico \% } \\
\text { ABS }^{\mathbf{a}}\end{array}$ \\
\hline 1 & $\mathrm{C}_{12} \mathrm{H}_{14} \mathrm{O}_{5}$ & 238.24 & 2.28 & 94.83 & 5 & 3 & 4 & 212.79 & 0 & 76.28365 \\
\hline 2 & $\mathrm{C}_{14} \mathrm{H}_{18} \mathrm{O}_{5}$ & 266.29 & 3.40 & 94.83 & 5 & 3 & 6 & 246.39 & 0 & 76.28365 \\
\hline 3 & $\mathrm{C}_{14} \mathrm{H}_{18} \mathrm{O}_{5}$ & 266.29 & 2.77 & 94.83 & 5 & 3 & 4 & 245.96 & 0 & 76.28365 \\
\hline 4 & $\mathrm{C}_{16} \mathrm{H}_{22} \mathrm{O}_{5}$ & 294.35 & 4.41 & 94.83 & 5 & 3 & 8 & 280.00 & 0 & 76.28365 \\
\hline tHGA & $\mathrm{C}_{19} \mathrm{H}_{24} \mathrm{O}_{4}$ & 304.39 & $5.08^{*}$ & 77.75 & 4 & 3 & 6 & 307.31 & 1 & 88.07 \\
\hline $\begin{array}{l}\text { tHGA } \\
\text { analogues }\end{array}$ & $\mathrm{C}_{20} \mathrm{H}_{27} \mathrm{O}_{4}$ & 346.47 & $6.64^{*}$ & 77.75 & 4 & 3 & 9 & 348.97 & 1 & 88.07 \\
\hline
\end{tabular}

aIn silico \% ABS was calculated by equation: 109-[0.345 x TPSA], bTPSA: Topological polar surface area, cMi Log P: Logarithm of compound partition coefficient between $n$-octanol and water, dnON: number of hydrogen bond acceptor, enOHNH: number of hydrogen bond donor, fnrotb: number of rotatable bonds, *did not meet the criteria of Lipinski's rule of 5 
Table 3: Results of ADME prediction using PreAdmet webserver

\begin{tabular}{|c|c|c|c|c|c|c|c|}
\hline Compound & $\begin{array}{l}\text { Molecular } \\
\text { formula }\end{array}$ & $\mathbf{P P B}^{\mathbf{a}}$ & $\begin{array}{l}\text { BBB } \\
\left(C_{\text {brain }} / C_{\text {blood }}\right)^{b}\end{array}$ & HIA\% ${ }^{c}$ & Caco-2 (nm/sec) & $\operatorname{MDCK}^{\mathrm{d}}(\mathrm{nm} / \mathrm{sec})$ & Skin permeability \\
\hline 1 & $\mathrm{C}_{12} \mathrm{H}_{14} \mathrm{O}_{5}$ & 93.376 & 0.624 & 75.286 & 20.588 & 60.789 & -3.874 \\
\hline 2 & $\mathrm{C}_{14} \mathrm{H}_{18} \mathrm{O}_{5}$ & 99.724 & 1.260 & 79.472 & 2.181 & 41.486 & -3.267 \\
\hline 3 & $\mathrm{C}_{14} \mathrm{H}_{18} \mathrm{O}_{5}$ & 100.000 & 1.27175 & 79.429 & 20.735 & 45.807 & -3.270 \\
\hline 4 & $\mathrm{C}_{16} \mathrm{H}_{22} \mathrm{O}_{5}$ & 100.000 & 2.392 & 82.840 & 10.444 & 9.739 & -2.621 \\
\hline tHGA & $\mathrm{C}_{19} \mathrm{H}_{24} \mathrm{O}_{4}$ & 100.000 & 2.145 & 88.415 & 12.891 & 0.247 & -2.040 \\
\hline $\begin{array}{l}\text { tHGA } \\
\text { analogue }\end{array}$ & $\mathrm{C}_{20} \mathrm{H}_{27} \mathrm{O}_{4}$ & 100.000 & 5.8597 & 89.88 & 14.4797 & 0.285 & -1.559 \\
\hline
\end{tabular}

aPPB: Plasma protein binding, bBBB: Blood brain barrier, cHIA: Human intestinal absorption, ${ }^{\mathrm{d}}$ MDCK: Madin-Darby canine kidney

\section{DISCUSSION}

Docking results showed that the hydroxyl group on the phloroglucinol moiety of derivative 2 (fig. 2) formed 2 important hydrogen bonds with the surrounding amino acid residues $\mathrm{Gln} 495(\mathrm{O}-\mathrm{H}-\mathrm{O}-\mathrm{H})$ and Gln697 $(\mathrm{O}-\mathrm{H}-\mathrm{O}-\mathrm{H})$ with a distance of $1.96 \AA$ and $1.94 \AA$, respectively. The benzene ring of the phloroglucinol moiety, formed a $\pi$-sigma bond with Ile553 with a distance of $3.92 \AA$, and interacts with Leu 754 through $\pi$-alkyl interaction, with a distance of $3.92 \AA$. Both the acyl side chains on the benzene ring were also stabilized through

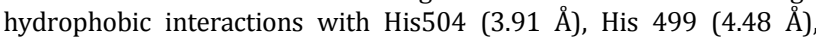

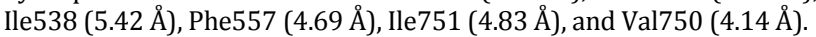

Similarly, the hydroxyl group on the benzene ring of derivative 4 (fig. 3 ) also formed $1.88 \AA$ and $2.00 \AA$ hydrogen bonds $(\mathrm{O}-\mathrm{H}-\mathrm{O}-\mathrm{H})$ with the same amino residues, Gln495 and Gln697. An unfavorable binding interaction detected between the hydroxyl group on the benzene ring and the nitrogen atom of $\operatorname{Trp} 500(\mathrm{O}-\mathrm{H}-\mathrm{N}-\mathrm{H})$ with a distance of $2.56 \AA$. The terminal end of the acyl chain formed van der Waals force with an amino residue, Ile553 (H-C-C-H) with a distance of $5.26 \AA$. Derivative 4 has comparative binding energy with the most active tHGA analogue (table 1). Derivative 4 has shorter side chains, consisting of two 5-Cs acyl side chains, while the latter consisted of one 5-Cs acyl group and one geranyl group. Derivatives 2 and 4 have hydrophobic interaction with the same amino acid

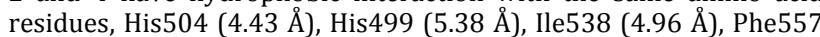
$\left(\begin{array}{ll}4.74 \AA\end{array}\right)$ and Val750 (8.95 $\left.\AA\right)$. Other residues involved in

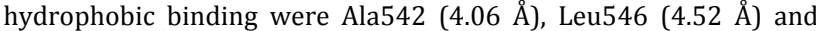
Ile552 (5.10 ̊̊).

As with derivatives 2 and 4 , the hydroxyl group on the phloroglucinol moiety of derivative 1 (fig. 4) was stabilized through hydrogen bonding with amino residues $\mathrm{Gln} 697$ (O-H-O-C), with distance of $2.04 \AA$ and with $\mathrm{Gln} 495(\mathrm{O}-\mathrm{H}-\mathrm{O}-\mathrm{C})$, with distance of $2.03 \AA$. Similar to derivative $2, \pi-\pi(\mathrm{H}-\mathrm{C}-\mathrm{C}-\mathrm{H})$, with distance of $5.47 \AA$, and $\pi$-alkyl $(\mathrm{H}-\mathrm{C}-\mathrm{C}-\mathrm{H})$, with distance of $5.06 \AA$, bonding interactions were observed between the benzene ring of derivative 1 with the amino acid residues Leu754 and Ile 553. The terminal end of the acyl chains also formed hydrophobic clusters with the residues Phe557 (4.94 ̊̊), Ile751 (4.78 Å), Val750 (3.89 Å), His504 (4.02 $)$ ), Ile538 (5.49 ̊) and His499 (4.54 ̊̊).

Derivative 3 (fig. 5) showed relatively different binding interactions, in comparison to all the other compounds. This could be due to its bulkier acyl side chain, which leads to more stearic hindrance and an altered binding affinity [23]. The hydroxyl group on the benzene ring of derivative 3 formed important hydrogen bondings with amino acid residues Gln579 (O-H-O-C), Asp584 (O-H-O-C) and Tyr493 (O-H-O-C), with distances of $2.06 \AA, 2.04 \AA$ and $1.93 \AA$, respectively. The carbonyl group of the acyl side chains also showed hydrogen binding interactions with residues Asn502 (C-O-H-N), with distance of $3.05 \AA$, and Gln $579(\mathrm{C}-\mathrm{O}-\mathrm{H}-\mathrm{N})$, with distance of $2.79 \AA$. The alkyl group on the acyl side chains interact with residue Trp574 (H-C-C-H) through $\pi$-alkyl bonding, with a distance of $4.42 \AA$. The amino acid residues involved in the hydrophobic binding interaction with the terminal end of the acyl side chains of derivative 3 included Leu689 (4.80 ̊), Leu501 (5.23 $)$ ) and Val570 (4.69 ̊).

Binding interactions observed for tHGA (fig. 6) and the most active tHGA analogue (fig. 7) were very similar. For example, both compounds are stabilized through similar hydrophobic binding interactions with amino acid residues Val570 and Trp574.
Furthermore, the hydroxyl and acyl groups of both compounds showed hydrogen bondings with amino acid residues Asp584 and Cys357. On the other hand, tHGA formed unfavorable bonding interaction, with a distance of $2.69 \AA$, between the hydroxyl group on phloroglucinol moiety with the amino acid residue Asp588 (O$\mathrm{H}-\mathrm{H}-\mathrm{N}$ )

In terms of molecular properties calculation, the molecular weights of all target compounds are within the limits of Lipinski rule (MWE $500 \mathrm{Da}$ ). This revealed that the diacyl derivatives, tHGA and tHGA analogue, have favorable permeability through cellular membranes. Meanwhile, all the diacyl derivatives (1-4) have LogP values of no more than 5, which were also within the limits of Lipinski's rule. The decreased $\log P$ values of the diacyl derivatives (1-4), as compared to tHGA and tHGA analogue, indicated that acylation improved aqueous solubility, which enabled the drug to transfers across membrane and be absorbed easily [21]. The low-aqueous solubility of prenylated acylphloroglucinols, due to significant lipophilicity, based on in silico study, have also been reported by Ilieve et al., (2018). Structural simplification, elimination of problematic functionalities, and decreased lipophilicity are important drug design strategies to improve the pharmacokinetic properties of the drug [22]. A mere reduction of 1 unit for $\log P$ value is equivalent to a 10 -fold increase in drug solubility [23]. Thus, it can be concluded that tHGA derivatives (1-4) can exhibit 10 to 30 -fold increase in solubility, based on the predicted value. The $\log P$ values of the diacyl derivatives (1-4) increased as the chain length of the acyl group increase; the LOX inhibitory activity increased throughout the series up to a maximum, corresponding to derivative 2 (4-C length; $7.26 \mathrm{kcal} / \mathrm{mol}$ ) and decreased to derivatives 4 (5-C length; $6.68 \mathrm{kcal} / \mathrm{mol}$ ) followed by derivative 1 (3-C length; $-6.37 \mathrm{kcal} / \mathrm{mol}$ ) and derivative 3 (4-C length with bulkier branched structure; $6.26 \mathrm{kcal} / \mathrm{mol}$ ).

The intermolecular interactions of a drug with water molecules can be altered by the number of hydrogen bond donors and acceptor groups within a molecular structure. With higher number of hydrogen bonds, it is more thermodynamically unfavorable for a drug to diffuse into a lipid environment, which means that it is more difficult for drug to transport across cellular membrane. As a result, the number of hydrogen bond groups is restricted in the Lipinski Rule of $5[24,25]$. The number of hydrogen bond donors (nOHNH) and number of hydrogen bond acceptors (nON) in tHGA compound, analogue and diacyl derivatives (1-4) obeyed the Lipinski's rule of 5.

The flexibility of the drug molecules, determined by the number of rotatable bonds within the drug structure, is used to determine the binding affinity of drug molecules with the receptor site. A flexible drug has a higher binding affinity compared to a less flexible drug [26]. All the target compounds evaluated in the present study have number of rotatable bonds which were in accordance with the Lipinski's rule i.e. less than 10. Therefore, all of the compounds are predicted to be orally active, in compliance with Lipinski's rule of 5 [27].

Topological polar surface area (TPSA) can be used to indicate the bioavailability of a drug compound as it is well correlated with the hydrogen bonding of the molecule. The TPSA values of tHGA and tHGA analogue were $77.75 \AA$, while the four diacyl derivatives were the same, i. e $94.83 \AA$, all of which did not exceed the limit of $160 \AA$. The percentage absorption values for the designed derivatives were $76.28 \%$, while for tHGA and tHGA analogue were $88.07 \%$. Generally, 
a compound is indicated as having good oral bioavailability if its percentage absorption ranged between $74.03 \%$ to $105.65 \%$. Based on the values shown by the target compounds, it was therefore predicted that all the compounds possess good oral bioavailability.

All the compounds also have Caco- 2 permeability values ranging from 10 to 20, except for derivative 2, indicating that they have moderate absorption in the human body (table 3 ). The low Caco-2 permeability value of 2.181 of derivative 2 suggested that the derivative possess low absorption potential. Based on MDCK values, drug permeability can be classified into three levels, i.e. low $(<25)$; medium (25-500) and high (>500). Derivatives 1,2 and 3 have medium permeability, while derivative 4 , tHGA compound and tHGA analogue have low permeability. Overall, the diacyl derivatives (1-4) showed better absorption than tHGA and tHGA analogue.

The skin permeability $(\mathrm{Kp})$ values for all the target compounds were negative (table 3). This indicated that they permeate the skin poorly [28]. These compounds were designed to be taken orally, therefore their Kp values should be low so that any accidental contact would not result in drug absorption. Human intestinal absorption (HIA) of a drug candidate is an essential property to evaluate for a potential drug candidate as it has significant influence over both bioavailability and absorption properties of the drug [29]. As shown in table 3, all the target compounds have HIA\% values of more than $70 \%$. Thus, the compounds are predicted to be well absorbed by the human intestine.

The percentage of plasma protein binding (PBB) is a critical pharmacokinetics factor in determining the optimum dosage regimen or frequency of administration [30]. All the tHGA derivatives presented more than $90 \%$ PPB value, which indicated that they are strongly bound to plasma protein. Therefore, higher dosages of the compounds could be applied. Blood-brain barrier (BBB) permeability is essential for drug molecules to be active in the central nervous system. As shown in table 3 , except for derivative 4 , all the designed derivatives exhibited moderate CNS absorption values. Similar to derivative 4, both tHGA and tHGA analogue exhibited high CNS absorption, thus they have unfavorable BBB properties.

\section{CONCLUSION}

The ligand-receptor binding interactions of diacyl derivatives of the natural product compound, tHGA, were studied through molecular docking. Derivative 2 showed promising LOX inhibitory activity, as indicated by its highest ligand-receptor interaction energies ($7.76 \mathrm{kcal} / \mathrm{mol}$ ). The presence of two acyl bearing groups result in a better pharmacokinetic profile, as shown by the in silico drug-like properties, predicted through Molinspiration and ADME profiling using PreADMET. The present study suggested that these tHGA derivatives could be promising compounds for further development into potent LOX inhibitors, which warrants further in vitro and in vivo investigations to confirm their pharmacokinetics and pharmacodynamics profiles.

\section{ACKNOWLEDGEMENT}

This study was funded by Management and Science University's seed grant (SG-042-022018-SPH).

\section{AUTHORS CONTRIBUTIONS}

All the authors have contributed equally.

\section{CONFLICT OF INTERESTS}

The authors declared that they have no conflicts of interest.

\section{REFERENCES}

1. WHO | Asthma; 2019. Available from: https://www.who.int/respiratory/asthma/en/. [Last accessed on 05 Nov 2020]

2. Radmark OP. The molecular biology and regulation of 5lipoxygenase. Am J Respir Crit 2000;161:S11-5.

3. Liu M, Yokomizo T. The role of leukotrienes in allergic diseases. Allergol Int 2015;64:17-26.

4. Tokuyama S, Nakamoto K. Pain as modified by polyunsaturated fatty acids. In: Omega-3 fatty acids in brain and neurological health. Academic Press; 2014. p. 131-46.
5. Eleftheriadis N, Poelman H, Leus NG, Honrath B, Neochoritis CG, Dolga A, et al. Design of a novel thiophene inhibitor of 15lipoxygenase-1 with both anti-inflammatory and neuroprotective properties. Eur J Med Chem 2016;122:786-801.

6. Wisastra R, Ghizzoni M, Boltjes A, Haisma HJ, Dekker FJ. Anacardic acid derived salicylates are inhibitors or activators of lipoxygenases. Bioorg Med Chem 2012;20:5027-32.

7. Dahlen SE. Treatment of asthma with antileukotrienes: first line or last resort therapy? Eur J Pharmacol 2006;533:40-56.

8. Gilbert NC, Barlett SG, Waight MT, Neau DB, Boeglin WE, Brash $\mathrm{AR}$, et al. The structure of human 5-lipoxygenase. Science 2011;331:217-9.

9. Skrzypczak Jankun E, Zhou K, Jankun J. Inhibition of lipoxygenase by (-)-epigallocatechin gallate: X-ray analysis at 2.1 Å reveals degradation of EGCG and shows soybean LOX-3 complex with EGC instead. Int J Mol Med 2003;12:415-20.

10. Shaari K, Suppaiah V, Lam KW, Stanlas J, Tejo BA, Israf DA, et al. Bioassay-guided identification of an anti-inflammatory prenylated acylphloroglucinol from Melicope ptelefolia and molecular insights into its interaction with 5-lipoxygenase. Bioorg Med Chem 2011;19:6340-7.

11. Ismail N, Jambari N, Zareen S, Akhtar M, Shaari K, Zamri Saad M, et al. A geranyl acetophenone targeting cysteinyl leukotriene synthesis prevents allergic airway inflammation in ovalbuminsensitized mice. Toxicol Appl Pharm 2012;259:257-62.

12. $\mathrm{Ng} \mathrm{CH}$, Rullah $\mathrm{K}$, Aluwi MFFM, Abas F, Lam KW, Ismail SI, et al. Synthesis and docking studies of 2,4,6-trihydroxy-3geranylacetophenone analogs as potential lipoxygenase inhibitor. Molecules 2014;19:11645-59.

13. $\mathrm{Ng} \mathrm{CH}$, Rullah K, Abas F, Lam KW, Ismail SI, Jamaludin F, et al. Hits-to-lead optimization of the natural compound 2,4,6trihydroxy-3-geranyl-acetophenone (tHGA) as a potent LOX inhibitor: synthesis, structure-activity relationship (SAR) study, and computational assignment. Molecules 2018;23:2509.

14. Mohamed MM, Jusril NA, Adenan MI, Ng KW. Molecular docking based on in silico screening of nci diversity sets for potent inhibitors of apobec3a enzyme. Int J Med Toxicol Forensic Med 2020;23:138-44.

15. Dirar AI, Waddad AY, Mohamed MA, Mohamed MS, Osman WJ, Mohammed MS, et al. In silico pharmacokinetics and molecular docking of three leads isolated from Tarconanthus camphoratus L. Int J Pharm Pharm 2016;8:71-7.

16. Ochieng PJ, Sumaryada T, Okun D. Molecular docking and pharmacokinetic prediction of herbal derivatives as maltaseglucoamylase inhibitor. Asian J Pharm Clin Res 2017;10:392-8.

17. Thakur A. Designing of potential new estrogen antagonists for treatment of Endometriosis: designing of ligands, molecular docking, activity, ADME and Toxicity prediction study. Int J Pharm Pharm 2013;5:451-5.

18. Banert K, Seifert J. Steric hindrance classified: treatment of isothiocyanatoallene with secondary amines bearing bulky substituents to generate 2-aminothiazoles. Org Chem Front 2019;6:3517-22.

19. Lipinski CA, Lombardo F, Dominy BW, Feeney PJ. Experimental and computational approaches to estimate solubility and permeability in drug discovery and development settings. Adv Drug Delivery Rev 1997;23:3-25.

20. Yamashita S, Furubayashi T, Kataoka M, Sakane T, Sezaki H, Tokuda H. Optimized conditions for prediction of intestinal drug permeability using caco-2 cells. Eur J Pharm Sci 2000;10:195-204.

21. Van de Waterbeemd H, Smith DA, Beaumont K, Walker DK. Property-based design: optimization of drug absorption and pharmacokinetics. J Med Chem 2001;44:1313-33.

22. Ilieva Y, Kokanova Nedialkova Z, Nedialkov P, Momekov G. In silico ADME and drug-likeness evaluation of a series of cytotoxic polyprenylated acylphloroglucinols, isolated from Hypericum annulatum morris subsp. annulatum. Bulg Chem Commun 2018;50:193-9.

23. Walker MA. Novel tactics for designing water-soluble molecules in drug discovery. Expert Opin Drug Discovery 2014;9:1421-33.

24. Clark DE. Rapid calculation of polar molecular surface area and its application to the prediction of transport phenomena. 2. Prediction of bloodbrain barrier penetration. J Pharm Sci 1999;88:807-14. 
25. Veber DF, Johnson SR, Cheng HY, Smith BR, Ward KW, Kopple KD. Molecular properties that influence the oral bioavailability of drug candidates. J Med Chem 2002;45:2615-23.

26. Feher M, Schmidt JM. Property distributions: differences between drugs, natural products, and molecules from combinatorial chemistry. J Chem Inf Comput Sci 2003;43:21827.

27. Haupt VI, Daminelli S, Schroeder M. Drug promiscuity in PDB: protein binding site similarity is key. PLoS One 2013;8:e65894.
28. Kumar K, R Giri A, Nadendla RR. In silico ADME profiling of CDK9 inhibitors. J Sci Res Pharm 2018;7:30-4.

29. Zhao YH, Le J, Abraham MH, Hersey A, Eddershaw PJ, Luscombe $\mathrm{CN}$, et al. Evaluation of human intestinal absorption data and subsequent derivation of a quantitative structure-activity relationship (QSAR) with the Abraham descriptors. J Pharm Sci 2001;90:749-84.

30. Bohnert T, Gan L. Plasma protein binding: from discovery to development. J Pharm Sci 2013;102:2953-94. 